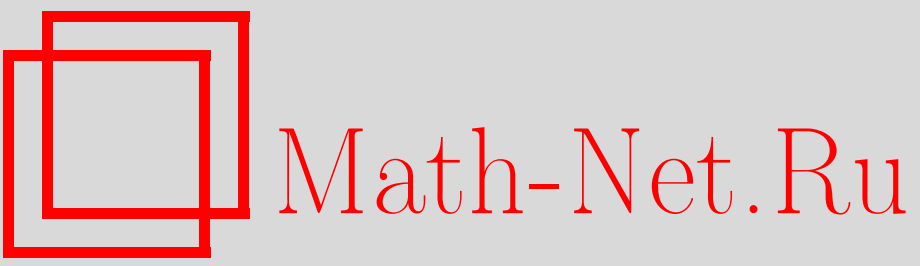

С. В. Сидоренко, О формулах регуляризованных следов, УМН, 1999, том 54, выпуск 5, 173-174

DOI: https://doi.org/10.4213/rm219

Использование Общероссийского математического портала Math-Net.Ru подразумевает, что вы прочитали и согласны с пользовательским соглашением

http://www.mathnet.ru/rus/agreement

Параметры загрузки:

IP : 18.209 .158 .208

26 апреля 2023 г., 14:28:12 


\title{
О ФОРМУЛАХ РЕГУЛЯРИЗОВАННЫХ СЛЕДОВ
}

\author{
С. В. СИДОРЕНКО
}

Полученные И. М. Гельфандом и Б. М. Левитаном [1] формулы регуляризованных следов для обыкновенных дифференциальных операторов с граничными условиями Штурма-Лиувилля послужили источником разнообразных обобщений. Одно из них связано с получением формул регуляризованных следов для абстрактных линейных операторов и интенсивно развивалось в статьях В. А. Садовничего и его учеников [2]-[5]. К этому направлению исследований примыкает данная статья.

Пусть $H$ - комплексное гильбертово пространство и задан положительно определенный самосопряженный линейный дискретный оператор

$$
T: D(T) \subset H \rightarrow H .
$$

Его собственные значения $0<\lambda_{1} \leqslant \lambda_{2} \leqslant \ldots$ и (учитывающая их кратность) асимптотика

$$
\lambda_{n}=C_{0} n^{\alpha}+O\left(n^{\beta}\right), \quad n \geqslant 1,
$$

где $C_{0}>0, \alpha>1,0 \leqslant \beta \leqslant \alpha$. Рассматривается также случай асимптотики вида

$$
\lambda_{n}=C_{0} n^{\alpha}+o\left(n^{\alpha}\right) .
$$

Пусть $V$ - линейный оператор, принадлежащий банахову пространству $L(H)$ линейных ограниченных операторов, действующих в $H$. Рассмотрим линейный оператор вида

$$
T-V: D(T) \subset H \rightarrow H,
$$

где оператор $T$ считается невозмущенным оператором, а оператор $V$ - возмущением, которое может быть несамосопряженным оператором.

Представим спектр $\sigma(T)$ оператора $T$ (см., например, [6]) в виде объединения

$$
\sigma(T)=\bigcup_{k=1}^{\infty} \sigma_{k}
$$

взаимно непересекающихся конечных множеств $\sigma_{k}, k \geqslant 1$, причем это разбиение строится по наперед заданным числам $b>0, \rho \geqslant 1$. А именно, существует последовательность $\left(r_{n}\right)$ неотрицательных чисел со следующими свойствами:

$$
\begin{aligned}
b n^{\rho \alpha} \leqslant & r_{2 n-1}<r_{2 n} \leqslant b(n+1)^{\rho \alpha}, \quad n \geqslant 1, \quad r_{0}=0 ; \\
r_{2 n}-r_{2 n-1} \geqslant & C_{1} n^{\rho(\alpha-1)}\left[1+n^{1-\rho(\alpha-\beta)}\right]^{-1}, \quad n \geqslant 1 ; \\
& \left(r_{2 n-1}, r_{2 n}\right) \cap \sigma(T)=\varnothing ; \\
\sigma_{k}= & \sigma(T) \cap\left[r_{2 k-2}, r_{2 k-1}\right], \quad k \geqslant 1 .
\end{aligned}
$$

Число собственных значений (с учетом кратности) $T$, лежаших в $\sigma_{k}$, не превосходит числа

$$
C_{2} k^{\rho-1}\left(1+k^{1-\rho(\alpha-\beta)}\right) .
$$

Рассматриваются следующие последовательности ортопроекторов: $P_{i}-$ проектор на собственное подпространство оператора $T$, отвечающее собственному значению $\lambda_{i}$;

$$
\mathscr{P}_{k}=\sum_{\lambda_{i} \in \sigma_{k}} P_{i}, \quad \mathscr{P}_{(n)}=\sum_{i=1}^{n} \mathscr{P}_{i}, \quad \mathscr{P}^{(n)}=I-\mathscr{P}_{(n)},
$$

где $I$ - тождественный оператор в $H$. Таким образом, введенные проекторы являются проекторами Рисса, построенными соответственно по спектральным множествам

$$
\sigma_{k}, \quad \Delta_{n}=\bigcup_{k=1}^{n} \sigma_{k}, \quad \sigma(T) \backslash \Delta_{n} .
$$

Подпространства, являющиеся образами соответствующих проекторов, обозначим

$$
H_{i}=\operatorname{Im} P_{i}, \quad \mathscr{H}_{k}=\operatorname{Im} \mathscr{P}_{k}, \quad \mathscr{H}_{(n)}=\operatorname{Im} \mathscr{P}_{(n)}, \quad \mathscr{H}^{(n)}=\operatorname{Im} \mathscr{P}^{(n)} .
$$


Введем в рассмотрение нелинейные операторы $\Phi_{n}: L(H) \rightarrow L(H), n \geqslant 1$, действующие в $L(H)$ и определенные равенствами

$$
\begin{aligned}
\Phi_{n}(X)=\sum_{\lambda_{i} \in \Delta_{n}}\left(V P_{i} X\right. & S_{n}\left(\lambda_{i}\right)-V S_{n}\left(\lambda_{i}\right) X P_{i} \\
& \left.\quad-P_{i} X S_{n}\left(\lambda_{i}\right) X \mathscr{P}^{(n)}+S_{n}\left(\lambda_{i}\right) X P_{i} X \mathscr{P}_{(n)}\right)+V, \quad X \in L(H) .
\end{aligned}
$$

Ограниченные операторы $S_{n}\left(\lambda_{i}\right), \lambda_{i} \in \Delta_{n}$, однозначно определяются из равенств

$$
S_{n}\left(\lambda_{i}\right)\left(\lambda_{i} I-T\right)=\left(\lambda_{i} T-I\right) S_{n}\left(\lambda_{i}\right)=\mathscr{P}^{(n)}, \quad \lambda_{i} \in \Delta_{n},
$$

т.е. каждый из таких операторов имеет вид: $S_{n}\left(\lambda_{i}\right) x=0$ на $\mathscr{H}_{(n)}$ и $S_{n}\left(\lambda_{i}\right) x=\left(\lambda_{i}-\lambda_{k}\right)^{-1} x$, если $x \in H_{k}, \lambda_{k} \in \sigma(T) \backslash \Delta_{n}$ (учитьвается, что $\left.H=\mathscr{H}_{(n)} \oplus\left(\bigoplus_{p \geqslant n+1} \mathscr{H}\right)\right)$.

Всюду считается, что число $\rho \geqslant 1$ выбрано так, чтобы

$$
\tau=\rho(\alpha-1)-\max \{0,1-\rho(\alpha-\beta)\}>0 .
$$

ЛЕмма. Пусть натуральное число $n_{0}$ въбрано из условия $\varepsilon_{n}=6 C_{1}^{-1}\|V\| n^{-\tau}<1$ $\forall n>n_{0}$, где постоянная $C_{1}$ взята из оченки (4).

Тогда каждий из операторов $\Phi_{n}, n>n_{0}$, переводит иар $\{X \in L(H):\|X\| \leqslant 2\|V\|\}$ в себя и является на нем оператором сжатия с константой сжатия $\varepsilon_{n}$. Последовательность операторов

$$
V_{k, n}=\Phi_{n}^{k}(0), \quad k \geqslant 0, \quad \Phi_{n}^{0}(0)=0
$$

сходится в $L(H)$ к некоторому оператору $X_{0, n} \in L(H)$ и имеют место оценки

$$
\left\|X_{0, n}-V_{k, n}\right\| \leqslant \frac{\varepsilon_{n}^{k}}{1-\varepsilon_{n}}\|V\|, \quad k \geqslant 0 .
$$

Оператор $T-V$ является дискретным оператором. Пронумеруем его собственные значения (с учетом алгебраической кратности) в порядке неубывания их вещественных частей. Получившуюся последовательность обозначим $\left(\mu_{k}\right)$.

Tеорема 1. Спектр сужения оператора $T-\mathscr{P}_{(n)} X_{0, n} \mathscr{P}_{(n)}$ на подпространство $\mathscr{H}_{(n)}$ совпадает с множеством $\widetilde{\Delta}_{n}=\left\{\mu_{1}, \ldots, \mu_{k}\right\}, k=\operatorname{dim} \mathscr{H}_{(n)}$.

Теорема 2. Пусть числа $\alpha, \beta, \rho$ и натуральные числа $m, N$ удовлетворяют одному из следующих условий

1) $\alpha-\beta \geqslant 1, N+1>\frac{m \alpha-1}{\alpha-1}, \rho=1$;

2) $\alpha>\beta, \alpha-\beta<1, N+1>\frac{m \alpha-\alpha+\beta}{\alpha-1}, \rho=\frac{1}{\alpha-\beta}$;

3) $\alpha=\beta, N+1>\frac{m \alpha}{\alpha-1}, \rho-$ любое;

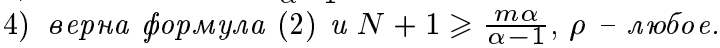

Тогда имеет место следующая формула регуляризованных следов:

(5) $\lim _{n \rightarrow \infty} \sum_{\lambda_{j} \in \Delta_{n}}\left(\mu_{j}^{m}-\lambda_{j}^{m}\right)+\operatorname{tr}\left[\left(T \mathscr{P}_{(n)}-\mathscr{P}_{(n)} \Phi_{n}^{N}(0) \mathscr{P}_{(n)}\right)^{m}-\left(T \mathscr{P}_{(n)}\right)^{m}\right]=0$.

\section{СПИСОК ЛИТЕРАТУРЫ}

[1] Гельфанд И. М., Левитан Б. М. // Докл. АН СССР. 1953. Т. 88. №4. С. 593-596. [2] Садовничий В.А., Дубровский В. В. // Дифференц. уравнения. 1977. Т. 13. № 11. С. 1264-1271. [3] Садовничий В. А., Любишкин В. А. // Докл. АН СССР. 1981. Т. 261. № 2. С. 290-293. [4] Садовничий В. А., Дубровский В. В., Любишкин В. А. // Докл. АН СССР. 1982. Т. 264. № 4. С. 830-832. [5] Дубровский В. В., Печенцов А. С. // Дифференц. уравнения. 1993. Т. 29. №1. С. 50-53. [6] Войтович Н. Н., Кацеленбаум Б. З., Сивов А.Н. // Обобшенный метод собственных колебаний в теории дифракции. М.: Наука, 1977. C. $289-416$. 